2009-07

\title{
A modular planar robotic manipulandum with end-point torque control
}

Howard, lan

http://hdl.handle.net/10026.1/4793

10.1016/j.jneumeth.2009.05.005

Journal of Neuroscience Methods

Elsevier BV

All content in PEARL is protected by copyright law. Author manuscripts are made available in accordance with publisher policies. Please cite only the published version using the details provided on the item record or document. In the absence of an open licence (e.g. Creative Commons), permissions for further reuse of content should be sought from the publisher or author. 


\title{
A modular planar robotic manipulandum with end-point torque control
}

\author{
Ian S. Howard ${ }^{*}, 1$, James N. Ingram ${ }^{1}$, Daniel M. Wolpert \\ Computational and Biological Learning Laboratory, Department of Engineering, University of Cambridge, Trumpington Street, Cambridge CB2 1PZ, UK
}

\section{A R T I C L E I N F O}

\section{Article history:}

Received 24 November 2008

Received in revised form 7 May 2009

Accepted 8 May 2009

\section{Keywords:}

vBOT

Robotic manipulandum

Motor learning

Torque

Stiffness

Object manipulation

Bimanual

\begin{abstract}
A B S T R A C T
Robotic manipulanda are extensively used in investigation of the motor control of human arm movements. They permit the application of translational forces to the arm based on its state and can be used to probe issues ranging from mechanisms of neural control to biomechanics. However, most current designs are optimized for studying either motor learning or stiffness. Even fewer include end-point torque control which is important for the simulation of objects and the study of tool use. Here we describe a modular, general purpose, two-dimensional planar manipulandum (vBOT) primarily optimized for dynamic learning paradigms. It employs a carbon fibre arm arranged as a parallelogram which is driven by motors via timing pulleys. The design minimizes the intrinsic dynamics of the manipulandum without active compensation. A novel variant of the design (WristBOT) can apply torques at the handle using an add-on cable drive mechanism. In a second variant (StiffBOT) a more rigid arm can be substituted and zero backlash belts can be used, making the StiffBOT more suitable for the study of stiffness. The three variants can be used with custom built display rigs, mounting, and air tables. We investigated the performance of the vBOT and its variants in terms of effective end-point mass, viscosity and stiffness. Finally we present an object manipulation task using the WristBOT. This demonstrates that subjects can perceive the orientation of the principal axis of an object based on haptic feedback arising from its rotational dynamics.
\end{abstract}

(C) 2009 Elsevier B.V. All rights reserved.

\section{Introduction}

\subsection{Background}

Over recent years many experiments investigating motor control have been carried out using robotic manipulanda. These often consist of a robotic arm with a handle attached to its end-point, which is held by a subject. Although such devices may be used as simple position tracking systems to investigate the kinematic properties of movements, they are frequently used in studies that involve dynamics. This can involve applying forces to the hand which depend on its state (such as its position or velocity). For example, velocity dependent curl fields are often used in the study of dynamic learning (Shadmehr and Mussa-Ivaldi, 1994). More sophisticated simulations may use a range of state-dependent forces to implement mass, viscosity, friction, and stiffness. Although many motor learning experiments have been carried out on a single arm, recently

\footnotetext{
* Corresponding author. Tel.: +44 (0)1223 748 520; fax: +44 (0)1223 332662 .

E-mail addresses: ish22@cam.ac.uk (I.S. Howard),jni20@cam.ac.uk (J.N. Ingram), wolpert@eng.cam.ac.uk (D.M. Wolpert).

1 These authors contributed equally to the work. ISH was responsible for the mechanical and electronic design. JNI was responsible for the software development and the object manipulation task. Both contributed equally to implementation and testing.
}

there has been interest in bimanual tasks which use pairs of manipulanda (Bays and Wolpert, 2006; Criscimagna-Hemminger et al., 2003; Howard et al., 2008; Tcheang et al., 2007). The study of arm stiffness is also an important issue in motor control. For example, when presented with unstable dynamics or tasks involving unpredictable forces, humans use muscle co-contraction to stiffen the limb and stabilize its movements. In such paradigms, manipulanda have been used to estimate limb stiffness (Burdet et al., 2001). In addition, manipulanda have been used to study object manipulation. However, due to limitations of available manipulanda, the object simulations in these previous studies were based on translational forces only. In contrast, real-world objects include rotational torques arising from their inertial properties (Kreifeldt and Chuang, 1979; Turvey, 1996).

\subsection{Existing two-dimensional manipulanda}

Many robotic manipulanda are documented in the literature and their design is usually optimized for the investigation of one specific aspect of motor control research (for example, dynamic learning or stiffness measurement). A large number of experiments can be adequately carried out using movements in two dimensions and most designs exploit this fact. Planar designs simply construction and offers performance benefits over those that must generate forces in all three extrinsic spatial dimensions. 
Most operate under impedance control, whereby the manipulandum reacts with the appropriate force for a given displacement. One of the first impedance controlled planar two-dimensional manipulanda designed to investigate human arm movements was developed at MIT (Faye, 1986). This uses a two-link serial arm similar in construction to a Selective Compliant Articulated Robot Arm (SCARA) design (Hiruma et al., 1999). Its 4-bar parallelogram structure gives the arm high stiffness. It is driven by two DC motors which are used to deliver joint torques independently at the manipulandum's shoulder and elbow. The motors are mounted coaxially, with one motor mounted above the arm and the other below. The inner link is driven directly whereas the outer link is driven via another bar running parallel to the inner link. Position measurements are made at the shoulder and elbow joint using absolute optical encoders and velocity measurements are calculated from optical incremental encoders. The motors are powered using switching servo amplifiers under computer control. A similar manipulandum, the MIT-Manus, was later commercialized for application in the rehabilitation of stroke patients (Hogan and Krebs, 2004; Hogan et al., 1992). The John Hopkins manipulandum is another two-degree of freedom device based on the mechanical design principles of the manipulanda described above (Charnnarong, 1991; Faye, 1986). A somewhat different approach was adopted in another MIT manipulandum that was a direct drive design using a gimbal consisting of a spherical two-degree of freedom linkage instead of the more classical arm parallelogram (Adelstein, 1989). Consequently the resulting motion of the endpoint traces out the surface of a sphere and does not remain within a plane. The configuration results in a very stiff mechanism and a variant of this design incorporating powerful motors has also been used in the estimation of arm stiffness (Franklin and Milner, 2003).

\subsection{Existing three-dimensional manipulanda}

Although planar designs are usually sufficient for many motor control paradigms, there are several manipulanda with three spatial degrees of freedom. Perhaps the best known are the Phantoms, originally developed at MIT and available in a range of sizes (Massie and Salisbury, 1994). They use light weight aluminium and carbon fibre arm sections powered by small DC motors via tensioned cable capstan drives to increase the mechanical advantage. The motors are arranged so as to decouple them from one another as much as possible, minimizing back-drive friction and inertia. An elegant feature of this design is that the motors themselves counterbalance the weight of the arm. These manipulanda were initially developed for use in virtual reality environments. As such they are limited in their force output and stiffness characteristics compared to the more robust planar designs, but are still widely used in the investigation of human motor control. The Phantom is also available with end-point torque control facility, although its rotational range and torque output is limited.

\subsection{Existing joint torque control manipulanda}

The manipulanda described above have a handle that must be firmly held by the subject and, as such, force is applied to the hand. In contrast some manipulanda have been designed to apply torques directly to the joints (Schouten et al., 2006; Scott, 1999). The Kinarm (Scott, 1999) can disassociate movements around the elbow with those around the shoulder, which cannot be achieved by simply applying force at the hand since the latter affects both joints. In addition, by applying the appropriate torques based on the configuration of the arm, the Kinarm can also simulate end-point force. For example, it can simulate viscous force fields in extrinsic space by applying the corresponding torques in joint space. It uses a 4- bar linkage that has hinged joints which can be aligned with the subject's shoulder and elbow joints. The subject's arm (human or monkey) is attached to the linkages using fibreglass braces. Two torque motors, fitted with encoders and torque sensors, drive their respective joints via timing belts.

\subsection{Existing manipulanda for stiffness measurement}

Several manipulanda have been developed specifically to measure stiffness. One such manipulandum was designed at Case Western Reserve University (Acosta et al., 2000). This is another two-dimensional planar device which can exert large forces $(220 \mathrm{~N})$ at its end-point and generate perturbations at relatively high frequencies. It achieves high end-point stiffness by using a fibreglass composite arm construction. Each joint is driven via cables from two large and powerful DC servo motors. Rotary encoders are mounted on the joints and a force transducer and an accelerometer are attached at the end-point. Closed-loop end-point velocity and position control is implemented using a dedicated digital signal processor. Another planar manipulandum built to measure stiffness is the ATR parallel-link direct drive air-magnet floating manipulandum or PFM (Gomi and Kawato, 1996, 1997). The design consists of two thin links driven by two wide links. The latter are directly driven from high power motors. The links are optimized for stiffness in the plane of movement and the handle is supported using a special frictionless magnetic-air bearing to prevent the links from bending downwards, which would result in movement out of the plane. The manipulandum is controlled using a dedicated digital signal processing unit.

\subsection{Existing manipulanda with active compensation}

A somewhat different approach to building a high performance manipulandum was adopted in the haptic master (Van der Linde et al., 2002). This is a three-dimensional design that operates using admittance control, whereby the user exerts a force on the device, which then reacts by generating the appropriate displacement. On the basis of measured end-point force, the controller actively compensates for the dynamics of the arm mechanism. The control software itself runs on a dedicated industrial PC. Using a real-time operating system it achieves a control loop rate of $2.5 \mathrm{kHz}$, which contributes to its high level of performance. Active compensation has the advantage that the manipulandum itself can be very powerful, stiff, and robust. It is a low backlash design which introduces friction at the joints, but this is fully compensated in the control software. Although this results in a system with high stiffness and virtually no friction, it is not ideal for dynamic learning because it still exhibits high residual end-point mass of around $2 \mathrm{~kg}$. In contrast, its characteristics make it ideally suited to simulations of stiff or massive objects. The issue of admittance control is also discussed at length by de Vlugt et al. (2003) in their design of a two-degree of freedom haptic device constructed to study the mechanical properties of the human arm.

\subsection{Aims}

Currently no single available manipulandum constitutes a general purpose tool for motor control research. For example, in dynamic learning paradigms which operate under force control, it is important that the intrinsic dynamics of the manipulandum are minimized so that they do not contribute excessively to the forces experienced by the subject. Although in practice such intrinsic dynamics cannot be eliminated completely, by careful mechanical design or by using active compensation, they can be kept as low as possible. In contrast, measuring arm stiffness places different constraints on the design of a manipulandum because this requires 


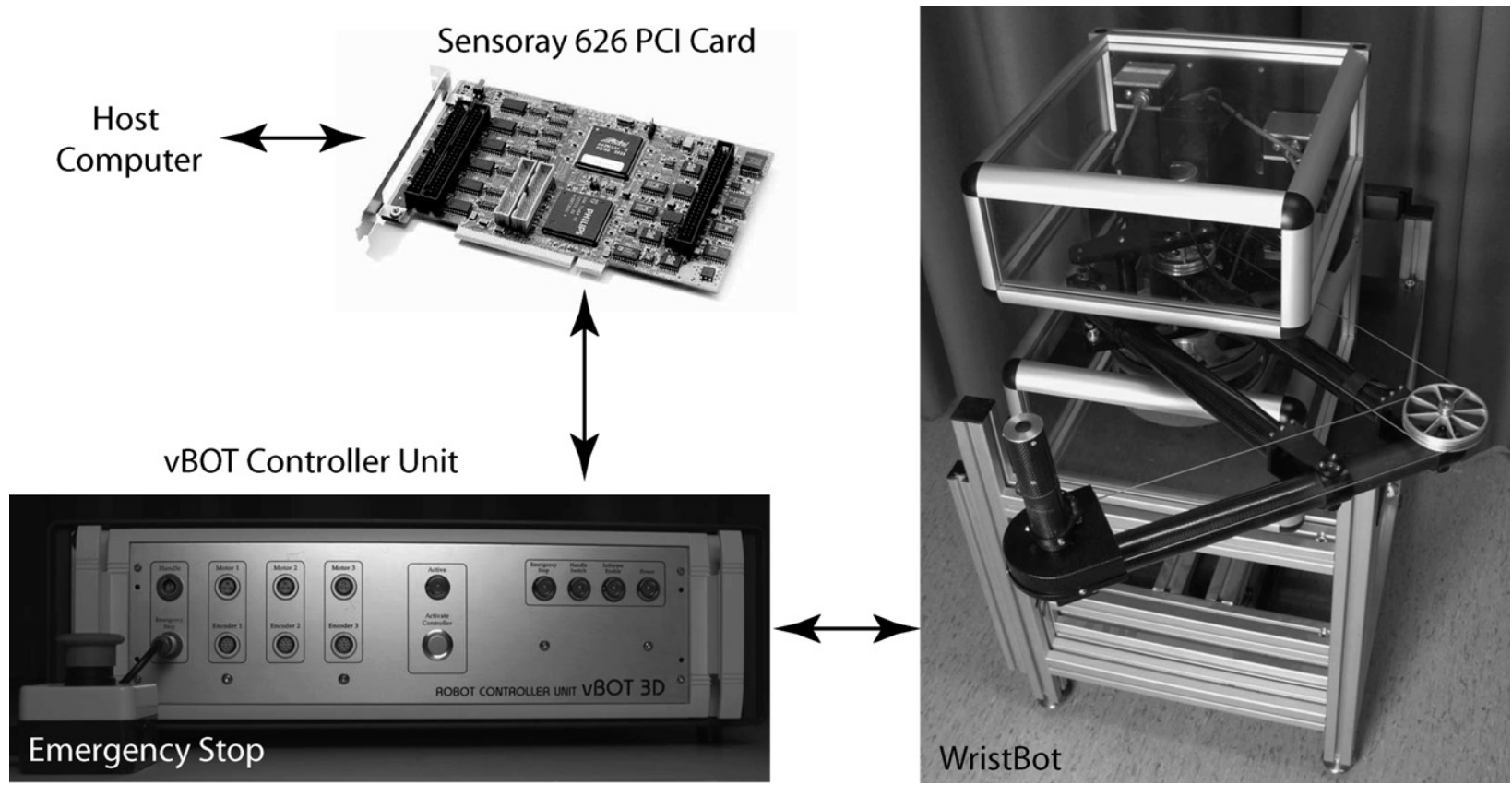

Fig. 1. The components of a vBOT system, showing the vBOT manipulandum, controller unit, emergency stop, and interface card.

position and velocity control. In this case, it is important that the manipulandum is optimized for high stiffness and high end-point force. The requirements for minimizing the intrinsic dynamics on the one hand and providing high force and stiffness on the other are thus at odds with each other. In addition, very few manipulanda have end-point torque control, which would be highly desirable for studying object manipulation. Together, these three points provided us with the incentive to build a modular manipulandum that would satisfy the diverse and potentially conflicting design requirements.

\section{Methods}

\section{1. $v B O T$ overview}

We now describe the generic planar two-dimensional manipulandum design (virtual reality roBOT or "vBOT") that is first and foremost optimized for motor learning paradigms, in which the intrinsic dynamics of the manipulandum must be minimized. We chose not to perform active compensation because this requires either feedback control based on precise sensing of the end-point force or feedforward control based on an inverse model of the dynamics or a combination of both. Instead, we chose to keep the intrinsic dynamics as low as possible and so this became a major design criterion. However, with the addition of an optional six-axis force transducer (ATI nano 25) in the handle, active compensation could be performed if desired. Additional design criteria we considered included stiffness, safety, accuracy, and force output range. It was also necessary to ensure that two units could be used directly beside each other for bimanual paradigms. A further discussion of the design requirements and relevant issues is provided in the supplementary material.

The overall vBOT system is composed of several distinct parts as shown in Fig. 1. It consists of the manipulandum, a custom built controller which includes electronic circuitry to drive the motors and implement safety features, and a computer system to implement real-time control within a custom written software development environment. The mechanical layout of the vBOT manipulandum is shown in Fig. 2.

\section{2. $v B O T$ design}

We used a $30 \mathrm{~mm}$ diameter steel shaft for the principal axis of the manipulandum to ensure great rigidity. It is supported by two massive solid pillars which are attached at the top and bottom of the base plate. The base itself is constructed from a $25 \mathrm{~mm}$ thick plate and provides a rigid foundation for the manipulandum which can be bolted onto a mounting table using angle brackets.

We adopt a 4-bar parallelogram arm structure for the vBOT (Fig. 2). This configuration compares favourably with alternative designs such as double-parallelogram, double-kite or band drive (Roy and Whitcomb, 1999). The workspace that can be reached by an adult arm suggested an appropriate length for both links of $40 \mathrm{~cm}$. The vBOT dimensions and workspace are shown in Fig. 2B. The arm is constructed from four linkages. Three links (the upper and lower arm links and the drive arm link) are constructed from carbon fibre tubes. These consist of epoxy bonded M60J fibres (TriCast Composite Tubes Ltd., Rochdale, UK) to achieve a very high stiffness and have an outside diameter of $40.1 \mathrm{~mm}$ and wall thickness of $1 \mathrm{~mm}$. The drive fork link completes the parallelogram by connecting the drive arm link to the pulley. It is constructed from aluminium. Mounting blocks fitted into one end of the upper arm and drive links are bolted onto the main and secondary drive blocks, respectively. The main block is attached to the main shaft using a bush. The secondary block is mounted on a shaft supported by a fourth link. The distal ends of the main arm and drive links are fitted with smaller mounting blocks and support forks. These support forks incorporate miniature ball bearings and firmly hold the lower arm link in position. A mounting block is fitted into the distal end of the lower arm link providing at attachment point for the handle mechanism. All carbon fibre tubes are glued to their mounting blocks using epoxy resin. The upper arm link is driven by the main shaft, which is itself driven by a large timing pulley. The fourth link, which drives the lower arm via the drive link, can rotate around the main shaft using ball bearings and is directly attached to another timing pulley. Rubber buffers prevent the arm from exceeding its working range thus avoiding damage due to unwanted collisions.

A rotating handle assembly is attached to a mounting block at the end of the lower arm link. It can be fitted in four different orien- 
(A)
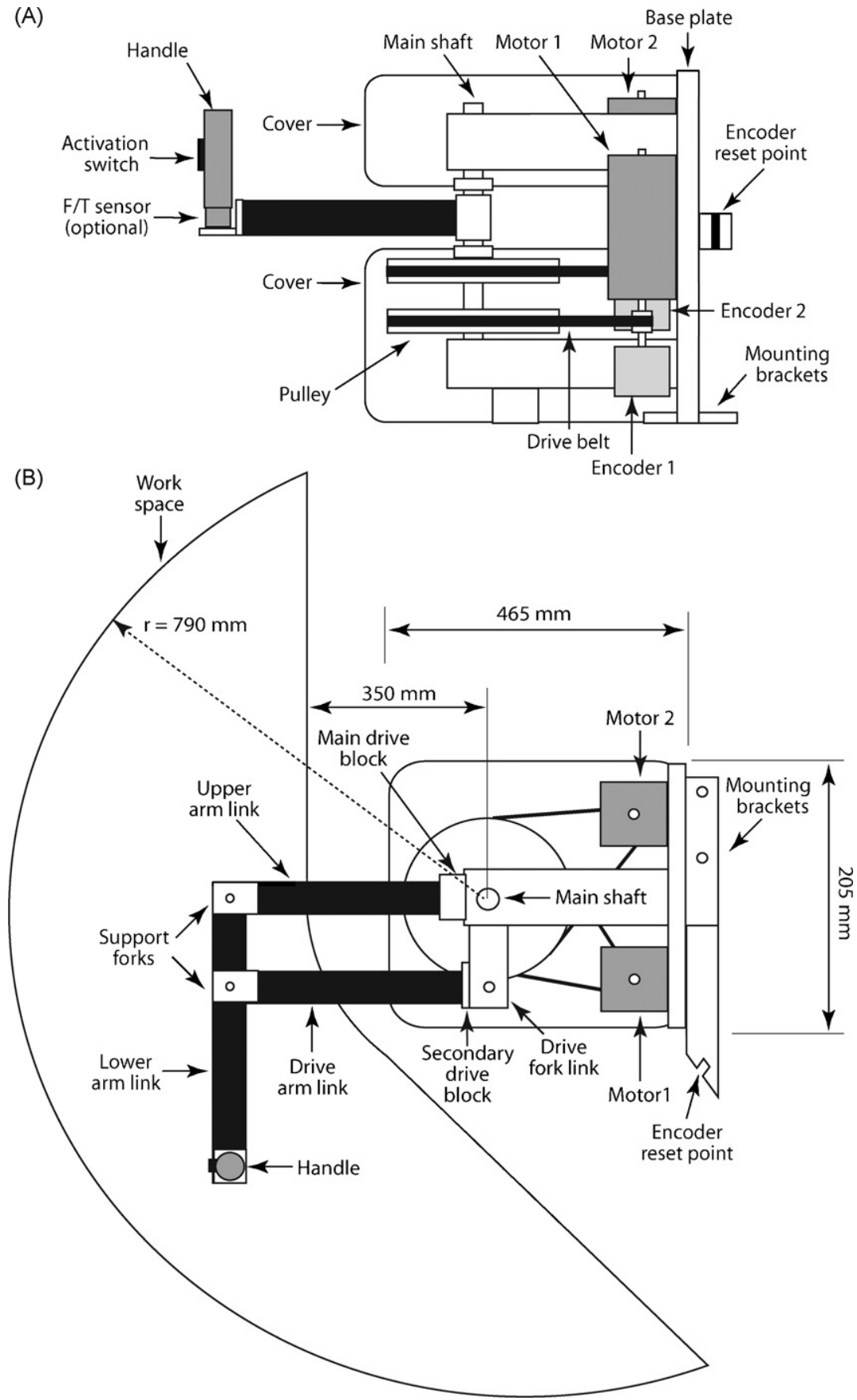

Fig. 2. Anatomy of standard vBOT manipulandum, showing major parts. (A) Side view. (B) A top view, including the workspace.

tations, but is usually inverted. In some circumstances it is desirable to directly measure the force exerted by the subject and provision is made for a force transducer at the base of the handle (Nano-25 sixaxis force/torque transducer, ATI Industrial Automation, Apex, NC, USA). The main body of the handle is constructed from $1.6 \mathrm{~mm}$ aluminium tubing, knurled on the outside to provide a good hand grip. It rotates around a central shaft using two precision ball races. The handle houses an activation switch as a safety feature, which prevents the manipulandum from generating forces unless it is firmly held by the subject. For bimanual setups, aluminium discs with a polyurethane rim can be fitted into the ends of the handles to prevent subjects banging their hands together.

Maxon rare earth DC motors (model RE75 118860) were chosen to drive the vBOT because they have a high torque constant of $0.233 \mathrm{~N} \mathrm{~m} / \mathrm{A}$, a high stall torque of $16 \mathrm{~N} \mathrm{~m}$ and a no-load speed of $1900 \mathrm{rpm}$ (Maxon Motor AG, CH-6072 Sachseln). Their ironless design exhibits a low moment of inertia and no cogging. They are designed to operate with Maxon switching amplifier controller units (ADS 50/10), which have a maximum continuous output current of $10 \mathrm{~A}$ continuous and maximum supply voltage of $50 \mathrm{~V}$. These amplifiers were the highest performance DC controllers available from Maxon at the time that the vBOT system was designed.

The drive mechanism employs a timing pulley transmission system to increase motor torque by a factor of seven (Transmission Development Co., Poole, Dorset, UK). Although timing belts can also introduce backlash, modern designs minimize this problem. The motors are mounted on the base plate in blocks, which are fitted into recessed channels so as to maintain their precise alignment. 
Each motor block assembly consists of a motor attached to a drive shaft. A small timing pulley (AL28-AT0/20-02) is fitted firmly to the shaft using a feather key. This small pulley drives a larger spoked timing pulley via a timing belt. All pulleys employed a "low backlash" tooth profile (a term used by the manufacturers) and friction was minimized by using a Teflon finish. The timing belts have a width of $25 \mathrm{~mm}$ which was necessary to withstand high tooth loading (25AT5/900 Generation 3 Synchroflex belts). Belt tension can be set using an adjustment mechanism to move the motor block back and forward in its recessed channel.

The far end of the motor drive shaft is supported by a self-aligning bearing and provides an attachment point for an incremental encoder. High-resolution encoders are used (Special Part Number: SP113347-30000, Industrial Encoders Direct Ltd., Wrexham, UK.). They operate up to speeds of $1000 \mathrm{rpm}$, employ shielded bearings to achieve low friction and generate $30 \mathrm{~K}$ pulses per revolution. This pulse count corresponds to a resolution in the order of $20 \mu \mathrm{m}$ at the handle. Because incremental position measurement is employed it is necessary to include an encoder reset point on the manipulandum and this is fixed to the rear of the base plate. Resetting the encoders involves positioning the handle at the reset point and zeroing the counters.

\subsection{WristBOT overview}

The standard vBOT design employs a handle that is free to rotate. A variant of this design, the WristBOT, can apply torque at the handle and measure its rotation. The design requirements for the WristBOT follow those of the standard vBOT. Firstly, the addition of rotational control of the handle should not compromise the performance of the existing translational mechanism. Secondly, the WristBOT mechanism itself should implement torque control with high stiffness and low levels of intrinsic dynamics.

The wrist drive allows handle rotations of up to $360^{\circ}$ with a maximum output torque of $3 \mathrm{~N} \mathrm{~m}$, which was considered suitable for most paradigms. This torque is the typical upper limit for torque screwdrivers that have a comparable handle size. To achieve this torque requires either a large direct drive motor or a smaller one employing a mechanical advantage. In either case the motor mechanism cannot be mounted at the handle itself because this would increase effective end-point mass unacceptably. Similarly, locating the motor drive in one of the arms is not an option because it would introduce significant anisotropic mass. We therefore chose to use a cable drive mechanism, with its heavy motor drive located at the rear of the vBOT base plate.

In this way, adding a cable driven handle to the standard vBOT design only involved changing the freely rotating handle for one that could be driven by a pulley at its base, and installing a cable drive mechanism. Since a cable can only pull, two cables are needed to rotate the handle in both directions. Although cables introduce some compliance into the drive mechanism, they have the advantage of only slightly increasing the effective end-point mass.

\subsection{WristBOT design}

The mechanical layout of the WristBOT is shown in Fig. 3. Flexible $1.2 \mathrm{~mm}$ diameter stainless steel cables are attached to the pulley at the handle and routed horizontally above the arm links and through holes in the base plate to the drive mechanism at the rear. The cables run via guide pulleys at the elbow and shoulder joints. The guide pulley at the elbow is simply fitted onto an extended joint shaft and rotates freely using miniature ball bearings. Similarly, the guide pulley at the shoulder rotates around the main manipulandum shaft using a $30 \mathrm{~mm}$ ball bearing. The handle is mounted directly onto the handle pulley which again rotates around a stiff shaft using precision ball bearings.
A helical thread matched to the cable diameter is cut into each pulley. The thread guides the cables and prevents them rubbing against each other as they move, ensuring low levels of friction. All pulleys are of the same diameter, so as they rotate the cables rise and fall equally on all pulleys, maintaining cable alignment throughout the drive system. A pulley diameter of $95 \mathrm{~mm}$ is used, representing a compromise between pulley mass and position accuracy. This issue arises due to stretch in the cables when loaded-smaller pulleys increase the effect of cable stretching, but have lower mass. Pulley moment of inertia is reduced by using a spoked construction.

Cables are attached to the handle and motor pulleys using cylindrical clamps. Care was taken to ensure that the location of the attachment points avoided direct stress on the clamps themselves. In fact, tension in the cable is never taken up by the clamp itself but rather by friction generated by contact between the cable and pulley over at least half its circumference. This is achieved by increasing the winding of the cable around each pulley above that needed to achieve the required rotational range. As a safety precaution the handle pulley is surrounded by a thin aluminium guard. To offset the additional mass of the torque transmission system, we replaced the standard vBOT handle with a much lighter design constructed from a carbon fibre tube.

For the sake of modularity, the motorized puller mechanism is built as an independent sub-assembly that is bolted to the rear of the base plate. Since the support structure does not move, it could once again be built in a sturdy fashion. Only the moment of inertia of its rotating parts had to be kept to a minimum. It employed a massive $25 \mathrm{~mm}$ thick base plate and two pillars to support an $8 \mathrm{~mm}$ drive shaft. The two cables are attached to separate drive pulleys which can be precisely aligned with the other pulleys in the mechanism. The drive pulleys are individually attached to the shaft using a bush, facilitating independent adjustment of the tension of each cable. Two motors drive the main shaft via a timing belt. A small pulley on the motors and a large pulley on the shaft provide a mechanical advantage of 3:1.

Two motors (Maxon special RE40 218010) were wired in parallel to double the available output torque. This achieves higher performance than a single larger Maxon motor (RE75) because it provides a similar peak torque $(2.14 \mathrm{~N} \mathrm{~m}$ for $2 \times$ RE40 versus $2.33 \mathrm{~N}$ m RE75) but with a lower total inertia (30\% that of the RE75). The motors are mounted in a small motor block that slides back and forth to allow the adjustment of belt tension. Measurement of angular position of the handle is made using a high-resolution incremental encoder attached to the back of one of the motors (SP113222-30000). This location is preferable to direct measurement at the end-point because it avoids adding moving mass. Similarly, it also realises close coupling between the encoder and the motors.

\subsection{StiffBOT overview}

The measurement of arm stiffness requires that the manipulandum itself be very stiff and operate under position control. Although the vBOT has been optimized for dynamic learning, stiffness can be increased with minimal modifications to the basic design. Most of the vBOT transmission is inherently stiff and the compliance arises predominately from the arm assembly. Consequently, stiffness can be increased by providing an interchangeable extra-stiff arm. This offers a choice between the lower effective end-point mass needed for motor learning paradigms and the higher end-point stiffness needed for impedance measurement.

\subsection{StiffBOT design}

Increased stiffness of the parallelogram arm was achieved by increasing the diameter of only the upper arm link from 40 to $50 \mathrm{~mm}$ diameter (Fig. 2). In addition, the carbon fibre wall thickness for 

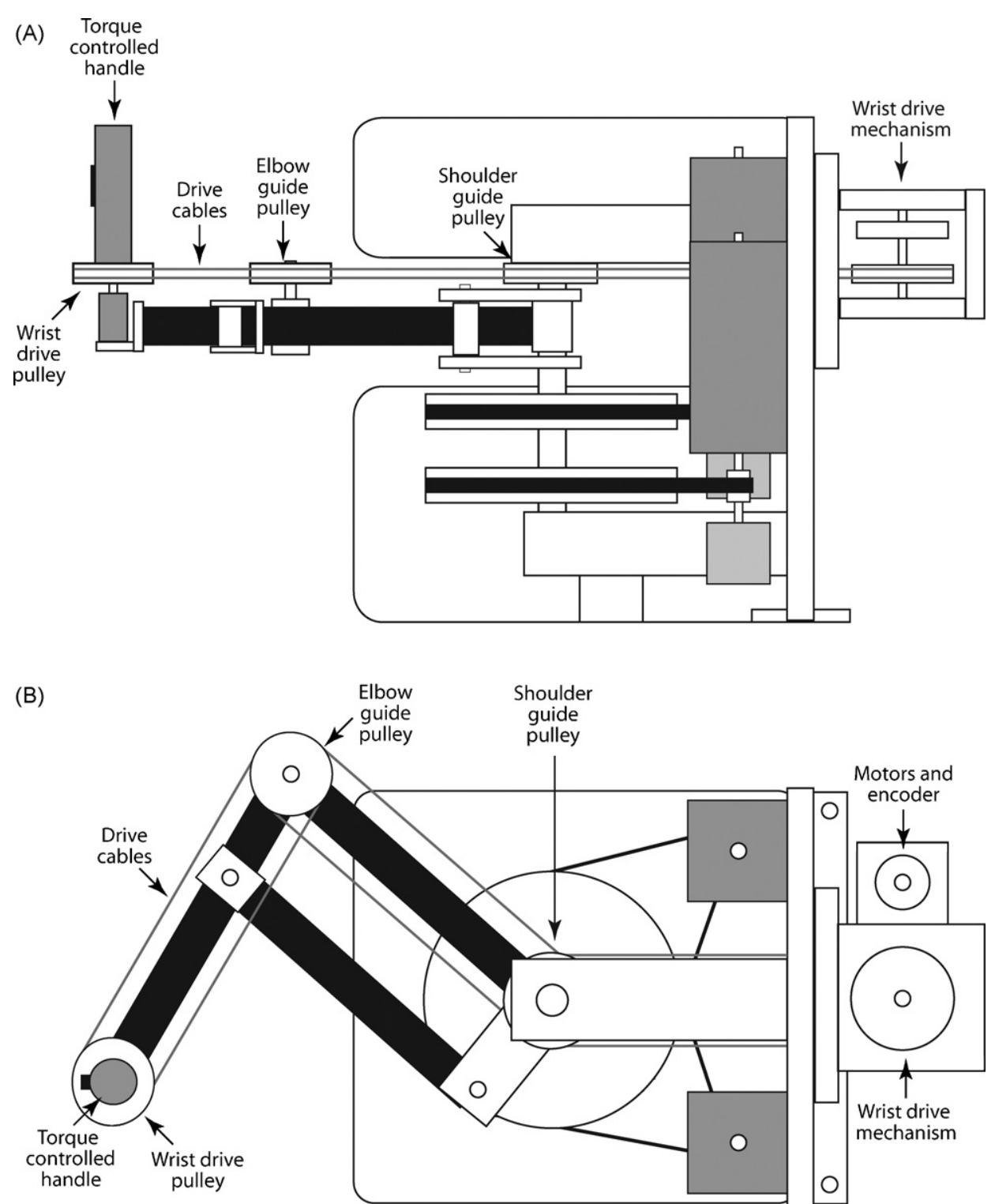

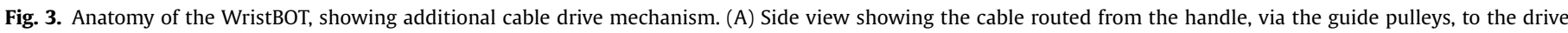
mechanism mounted on the base plate. (B) Top view.

both the upper and lower arm links was increased from 1 to $2 \mathrm{~mm}$. These changes increased the stiffness of the links of the arm itself (not of the entire vBOT system as measured at the handle) by a factor of approximately 3 at the cost of adding a few hundred grams to the effective end-point mass. To further increase stiffness the "low backlash" pulleys in the standard design were replaced with "zero backlash" pulleys (again manufacture's term to indicate that they were optimized to run at low speeds with zero play). Because the drive arm link does not bend, it remained unmodified.

\section{7. $v B O T$ controller}

A controller and PC are needed to operate the manipulandum (Fig. 1). The vBOT controller provides the interface between the hardware components of the manipulandum and a multi-function IO card within the computer. It provides drive for the motors and implements safety features, such as the emergency stop, handle activation switch and software enable.

The recommended controllers for the larger Maxon motors are switching amplifiers and consequently these formed the basis of the
vBOT controller unit. They are configured to control motors in current mode, whereby input voltage determines the output current and therefore motor torque. Three Maxon ADS 50/10 amplifier units are used in each vBOT controller, which can be used to drive either a standard vBOT or StiffBOT (with two motors) or a WristBOT (with three motors). These switching amplifiers require a high power DC supply and to avoid any possible interference effects with a switching regulator, a simple passive unregulated design was used. This consisted of a $500 \mathrm{~W}$ toroidal transformer, bridge rectifier and large smoothing capacitor.

To implement status indication and safety features, simple logic circuitry is implemented on a PCB. This circuitry ensures that the controller could not generate output to the motors until a software application on the host PC has written an appropriate bit pattern to the digital input on the controller unit. It also ensures that the motor controller does not activate until the handle switch is depressed. In addition there is a separate emergency stop circuit which will cut motor power when operated. This is a safety feature which allows the operator to rapidly disable the manipulandum in the event of unexpected forces. To implement this, output to the motors run via 
normally open relays. To close the relays and connect the controllers to the motors, an activate controller button on the front panel must be pressed. Pressing the emergency stop switch immediately opens the relays and disconnects the motors. This circuit includes a failsafe mechanism and automatically disconnects the motors in the event of power failure. In this case the system does not activate again until explicitly reactivated. The logic circuit also drives a set of status LEDs on the front panel of the controller.

The controller is built into a sturdy $19 \mathrm{in}$. rack-mounting case and employs a custom machined heavy duty base plate, providing a firm attachment point for the internal components. It also acts as an excellent heat sink. The status LEDs, and connectors for the motors, encoders, handle switch and emergency stop switch are mounted on the front panel. Ribbon cable connectors are mounted on the rear panel which connect to the multi-IO card in the host PC. The front and rear panels are custom built to professional standards (Fig. 1).

\subsection{Computer control}

An off-the-shelf multi-function input/output PCI card (model 626, Sensoray Co. Inc., Tigard, Oregon, USA; Fig. 1) provides the hardware interface between the host PC and the vBOT controller. The Sensoray 626 card includes digital-to-analogue (DAC) channels for analogue control of motor torque, digital input/output (DIO) channels for digital control of the status and activation circuits, incremental counters for reading the encoders and analogue-todigital (ADC) channels for reading analogue devices such as a force sensor. A single card provides sufficient channels to control two standard vBOTs (for example, a bimanual rig) or a single WristBOT. Multiple cards are supported for more elaborate configurations.

A software environment was developed to support the easy creation of application programs that implement experimental paradigms using the vBOT. This includes a software library and support for multiple and differently configured experimental rigs.

The library implements all basic operations needed to control the vBOT and hides the low level operations from the experimenter. All software is developed using Microsoft Visual C++ under Windows 2000/XP. Close-to-real-time performance is obtained by using the Windows Multimedia Timer, which allows a high-resolution timer event to be scheduled with a period of $1 \mathrm{~ms}$. During operation the scheduled timer event calls the vBOT control loop function and provides a software update rate of $1000 \mathrm{~Hz}$. However, because the operating system is not real-time, there is some jitter around the $1 \mathrm{~ms}$ update period. During a $10 \mathrm{~s}$ window, for example, the mean period on our test PC was $1.000 \pm 0.149$ ms (mean \pm SD). This value for the mean period suggests misleadingly good performance, because in many cases the distribution of the Multimedia Timer period is actually bimodal. On our test PC, for example, a timer event is missed approximately every $42 \mathrm{~ms}$, and consequently the period for the subsequent event is close to $2 \mathrm{~ms}$. Thus, to implement time-dependent algorithms, such as those required to differentiate encoder position to provide velocity and acceleration, the actual period must be determined for each iteration of the control loop. To do this, we use the Windows High-Resolution Counter, which gives a resolution on our test PC of less than $1 \mathrm{~ns}$. By combining the close-to-real-time performance provided by the Multimedia Timer with the accurate timing of events supported by the HighResolution Counter, it is possible to implement a suitable software control loop at $1000 \mathrm{~Hz}$.

\subsection{Machine guards}

Because high and potentially dangerous end-point speeds and force levels can be generated by the vBOT, user safety must be taken seriously. Therefore a physical guard is placed around the drive
(A)
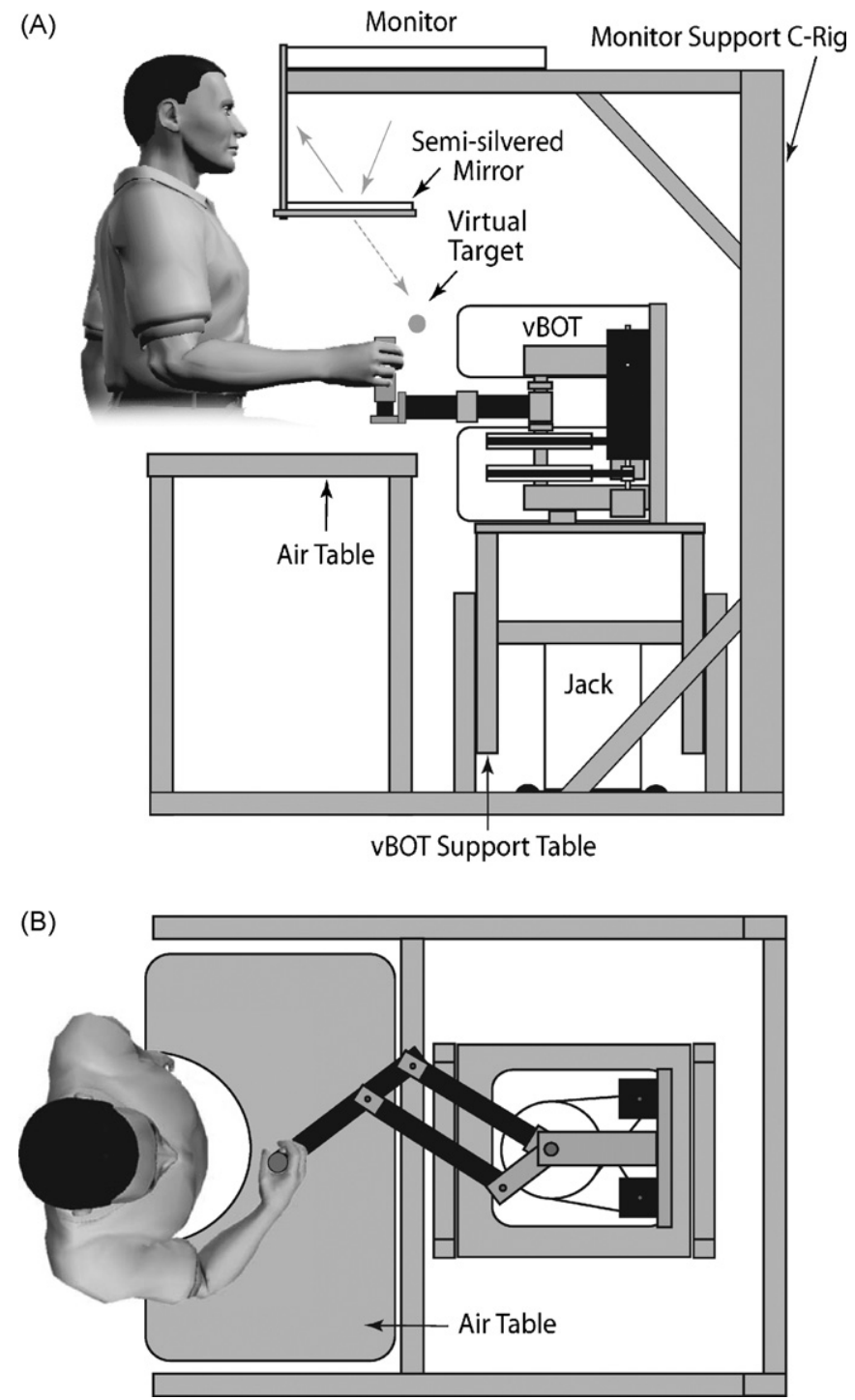

Fig. 4. The vBOT virtual reality system. (A) Side view showing the vBOT, its adjustable-height support table and the C-Rig which supports the monitor and semisilvered mirror. The C-Rig is designed to provide a large unobstructed workspace. The vBOT table incorporates a jack for height adjustment. (B) Plan view showing location of air table.

mechanisms to prevent access by hands and clothing. It is constructed from aluminium profile and half rounded sections are used at the front and sides. The vBOT arm protrudes through a horizontal gap between two sections which are fitted with clear polycarbonate windows to maintain visibility of the internal parts (Fig. 2). The subject should also be seated such that the handle cannot reach their body or head. This requirement is met implicitly when using the air table described below.

\subsection{Mounting table}

To run experiments while either sitting in a chair or standing, the manipulandum must be positioned at a height suitable for each subject (Fig. 4). Because the vBOT is very heavy, a stable adjustable mounting table is required to ensure it remains stationary during operation. The vBOT table is constructed from $50 \mathrm{~mm}$ square section aluminium profile to ensure high rigidity. The table consists of an inner cube fitted with a $15 \mathrm{~mm}$ thick aluminium table top. To permit the adjustment of table height it has two leg assemblies which move up and down on the outside of this cube on nylon runners fitted 
into slots in the profile. A screw jack is attached to the inner table to move it up and down. After the height has been adjusted, the assembly can be firmly secured using four knobs which clamp the legs to the inner table. The jack is also fitted with castors to assist with movement of the vBOT around the laboratory (Fig. 4).

\subsection{Virtual reality C-rig}

In the simplest case, a display monitor can be placed on top of the vBOT to provide visual feedback related to the experimental paradigm. However, it is more desirable to use a more sophisticated virtual reality environment that can overlay visual feedback in the plane of movement. This can be achieved by back-projecting an image onto a screen which is then viewed via a mirror located below it (Goodbody and Wolpert, 1998). By correctly positioning the mirror, the image can be made to appear at the location where the hand grasps the handle. If the mirror is semi-silvered, this allows calibration of the graphical workspace to that of the manipulandum. Due to recent developments in display technology, the screen and projector can now be replaced with a large computer display (for example, Apple 30" Cinema HD Display, Apple Computer, Cupertino, CA, USA). The main function of a C-rig is thus to firmly hold this large monitor and mirror assembly (Fig. 4). The rig accommodates two vBOTs (for bimanual studies) and an air table (see below).

The C-rig was designed to be stable so as to prevent movement of the image and to provide a large unobstructed workspace for the manipulanda. Thus care was taken to keep the rear and sides of the rig clear of any structural components. From the side the rig employs a $\mathrm{C}$ configuration giving it its name. This facilitates positioning of the subject, and easy accommodation of other equipment within the workspace. The rig is constructed from $40 \mathrm{~mm}$ section aluminium profile. Bracing sections set at $45^{\circ}$ are used to maintain high structural stability. The monitor support structure can be adjusted in height by sliding it up and down on the main rear support columns.

\subsection{Air sled table}

For some experimental paradigms, in particular in the measurement of stiffness, it is important to constrain arm movement to a plane. This can be achieved by using air sleds to support the arms during movements. Air sleds can be built from three air pucks, which achieve very low friction by floating on a cushion of air over a smooth surface. A custom built adjustable air table and air sleds were developed as part of the vBOT system. The main design requirement for the air sleds themselves is to ensure they contribute only slightly to the moving mass off the arm. The air pucks must align with the surface they float over, because poor alignment leads to air leakage and an increase in friction due to contact between the puck and table.

The vBOT air sled consists of three aluminium air pucks each with a $30 \mathrm{~mm}$ base diameter (Fig. 5). They are mounted at the vertices of an equilateral triangle using a spherical bearing to ensure alignment. Air connection to each puck is made at the side and air exits at its base through a $1 \mathrm{~mm}$ diameter hole. The triangle is constructed from three aluminium nodes held together by carbon fibre rods attached using epoxy resin. A carbon fibre arm rest is attached to the supporting triangle. The air pucks are connected to the air supply using $6 \mathrm{~mm}$ diameter polythene tubing. Care was taken to ensure the same tube airflow resistance was present for each puck. This was achieved by using T-joining sections such that each puck received air via a single $90^{\circ}$ joint (Fig. $5 \mathrm{C}$ ). The sled typically operates at 4 bar air pressure and when used on the smooth glass surface will support at least $20 \mathrm{~kg}$ with virtually zero friction. The sled itself weights only $160 \mathrm{~g}$.
A height adjustable air table is constructed from $40 \mathrm{~mm}$ section aluminium profile (Fig. 4B). To achieve the required height, the supporting legs could be slid up and down on the outside of an inner table section. They are then secured using four knobs to clamp them firmly. The table top consisted of a $10 \mathrm{~mm}$ thick toughened glass plate, providing a smooth surface ideal for use with the air sled. A region is cut out of the glass sheet so that subjects are surrounded on three sides by the table, providing a large workspace for both the left and right arms.

The presence of an air table also represents a safety feature of the vBOT because it maintains a safe distance between the subject and the manipulandum and ensures that the body of a subject is out of contact range of the handle.

\subsection{Performance evaluation of $v B O T$ and variants}

To quantify the performance of the vBOT manipulanda, a series of experiments were run to measure their physical characteristics in terms of static and dynamic friction, stiffness, effective end-point mass and viscosity at the handle (Hayward and Astley, 1996). All measurements were carried out on the standard vBOT. In addition mass and viscosity measurements were repeated on the StiffBOT and WristBOT. Stiffness measurements were only repeated on the StiffBOT since the WristBOT used the same basic arm components as the standard vBOT. In addition the frequency response of the StiffBOT was evaluated, which is of particular interest for manipulanda used to measure arm stiffness.

Static friction and stiffness measurements were made at a single central point in the vBOT workspace. Dynamic properties, which change with handle position, were measured at nine different positions across the workspace, as defined by the vertices of a grid with $15 \mathrm{~cm}$ spacing centred on the workspace origin.

To measure the maximum range of the workspace, the handle was moved by hand along the limits of its reach while position was recorded. Static friction was estimated by applying a slowly increasing linear force to the handle (by manually pulling it via an elastic band) and recording the force level at which it started to move. This was carried out at the central location over 30 trials. Static stiffness for both the vBOT and the StiffBOT was measured at the handle in the central workspace location by firmly attaching it to suitably massive object, that is, the rear of another vBOT, using a specially built clamp. This held the handle over approximately the top two-thirds of its length, distal to its attachment to the arm. The vBOT applied forces of $10 \mathrm{~N}$ in eight directions. The corresponding displacements due to the deflection of the arm and transmission mechanisms were recorded by the encoders. A stiffness ellipse was calculated as the least-squares solution from the force and deflection measurements.

Uncompensated closed-loop stiffness at the handle was also estimated at the central position in eight directions for the StiffBOT. A force transducer was attached to the handle to record force and a spring was simulated with six levels of stiffness $[10,20$, $\ldots, 60 \mathrm{~N} / \mathrm{cm}]$. The true handle position was measured using an optical position measurement system (Optotrak, Northern Digital Inc., Ontario, Canada) while the handle was manually pulled in all directions around the zero position of the simulated spring. The force and deflection at the handle were used to calculate the spring stiffness. This is the actual value of spring stiffness generated by the complete vBOT system without taking the compliance of the mechanism into account.

To estimate effective end-point mass, viscosity and dynamic friction of the vBOT, a force transducer was again attached to the handle. At a given location, the handle was manually pulled around via a piece of elastic attached to the force transducer. In total $60 \mathrm{~s}$ of data was recorded at nine workspace locations. Care was taken to collect a complete range of movements in all directions without 


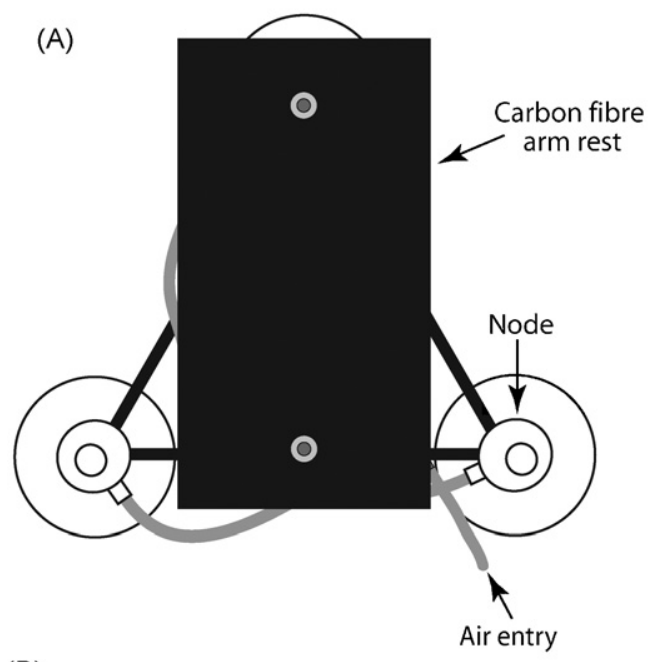

(B)

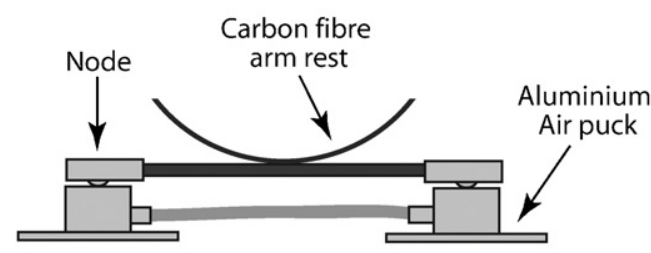

(C)

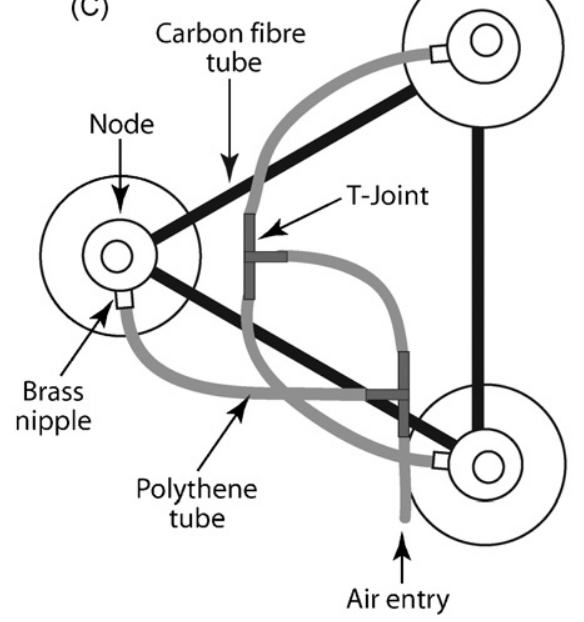

(D)

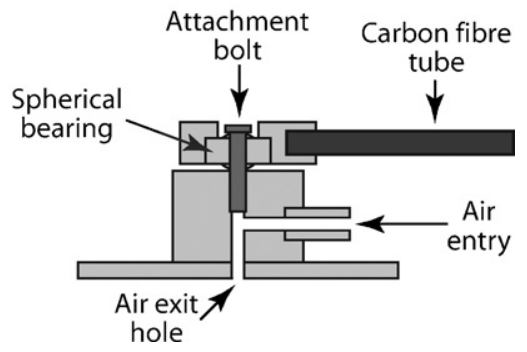

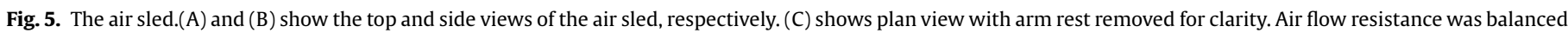

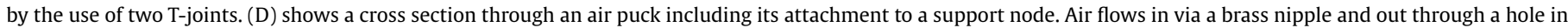
the base. A spherical bearing is used to connect the puck to its support node, allowing it to align with the surface of the air table.

deviating too far from the measurement location. Handle velocity and acceleration were numerically differentiated from the position signal which had been low-pass filtered using a zero-shift fifthorder Butterworth filter with an $8 \mathrm{~Hz}$ cut-off frequency. The 2D force vector $\boldsymbol{F}$ measured at the handle was modelled by the following equation:

$$
\left[\begin{array}{c}
\boldsymbol{F}_{x} \\
\boldsymbol{F}_{y}
\end{array}\right]=\left[\begin{array}{ll}
I_{x x} & I_{x y} \\
I_{y x} & I_{y y}
\end{array}\right]\left[\begin{array}{c}
\ddot{x} \\
\ddot{y}
\end{array}\right]+\left[\begin{array}{ll}
B_{x x} & B_{x y} \\
B_{y x} & B_{y y}
\end{array}\right]\left[\begin{array}{c}
\dot{x} \\
\dot{y}
\end{array}\right]+\left[\begin{array}{l}
\operatorname{sign}(\dot{x}) S_{x} \\
\operatorname{sign}(\dot{y}) S_{y}
\end{array}\right]
$$

At each measurement location effective end-point mass $I$, viscosity $B$ and dynamic friction $S$ were fitted to the data points using multiple linear regression (Perreault et al., 2002). High $r^{2}$ values $(>0.93$ ) showed a good fit to the data. The effective endpoint mass estimated in this way included a contribution due to the sensing-part of force transducer itself which was subtracted from the estimates.

The frequency response of the StiffBOT was measured by first clamping the end of its lower arm to a force transducer, which itself was attached to the rear of another vBOT. The StiffBOT was then driven sinusoidally to generate a peak-to-peak force of $4 \mathrm{~N}$. This was carried out at a range of frequencies between 1 and $80 \mathrm{~Hz}$ (frequencies shown in Fig. 7). In each case the force was generated for $4 \mathrm{~s}$. Spectral analysis was used to determine the amplitude and phase response at each driving frequency.

All analyses were conducted using Matlab (The MathWorks Inc., Natick, MA, USA).

\subsection{Object manipulation task}

When grasping and manipulating an object, the inertial properties give rise to forces and torques. Gibson (1966) suggested that the perception of these dynamic properties of objects, based on the forces and torques experienced during active object manipulation, should be regarded as a separate perceptual subsystem, which he called dynamic touch. Experiments documenting the perceptual abilities of dynamic touch in humans have traditionally involved subjects manipulating physical objects behind an opaque screen that occludes vision (Turvey, 1996). Such experiments have shown, for example, that humans can perceive the length (Solomon and Turvey, 1988) and orientation (Turvey et al., 1992) of occluded physical objects simply by wiggling them. Our motivation in developing the WristBOT, which adds a third rotational dimension to the standard vBOT, was to provide a system for studying object manipulation using virtual reality. Specifically, the WristBOT allows us to simulate the rotational dynamics of objects, which are thought to be especially important for the perceptual abilities associated with dynamic touch (Amazeen and Turvey, 1996; Turvey, 1996).

Subjects $(n=8)$ performed a haptic discrimination task which required them to indicate the direction to the centre of mass of a virtual object. Two of the subjects were authors (ISH and JNI) and the remainder were volunteers, naïve to the purpose of the experiment. All subjects gave written consent in accordance with the requirements of the local ethics committee. Subjects were seated and grasped the handle of the WristBOT with their right hand. The WristBOT simulated a rigid body of mass $m$ that could be rotated around an axis which was offset from the centre of mass by distance $d$ (Fig. 8A). The value of $m$ and $d$ were held constant at $1 \mathrm{~kg}$ and $7 \mathrm{~cm}$, respectively throughout the experiment. The rotation axis was fixed with regards to its distance from the centre of mass of the object, but it was otherwise free to translate with the object. Consequently, rotation generated both torques and translational forces.

Subjects completed a familiarization block of 50 trials followed by an experimental block of 50 trials. On each trial the direction of 

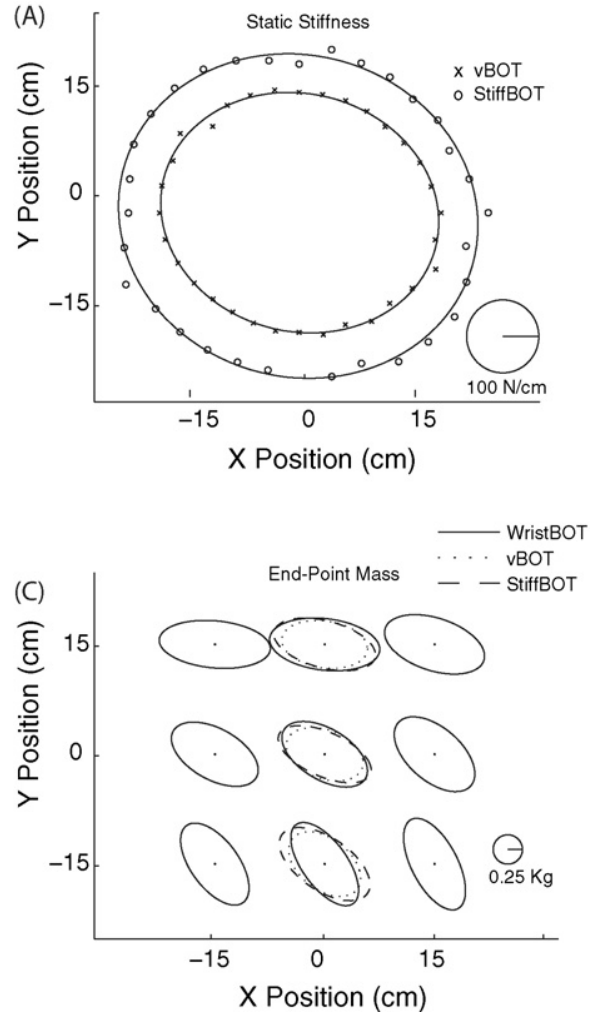
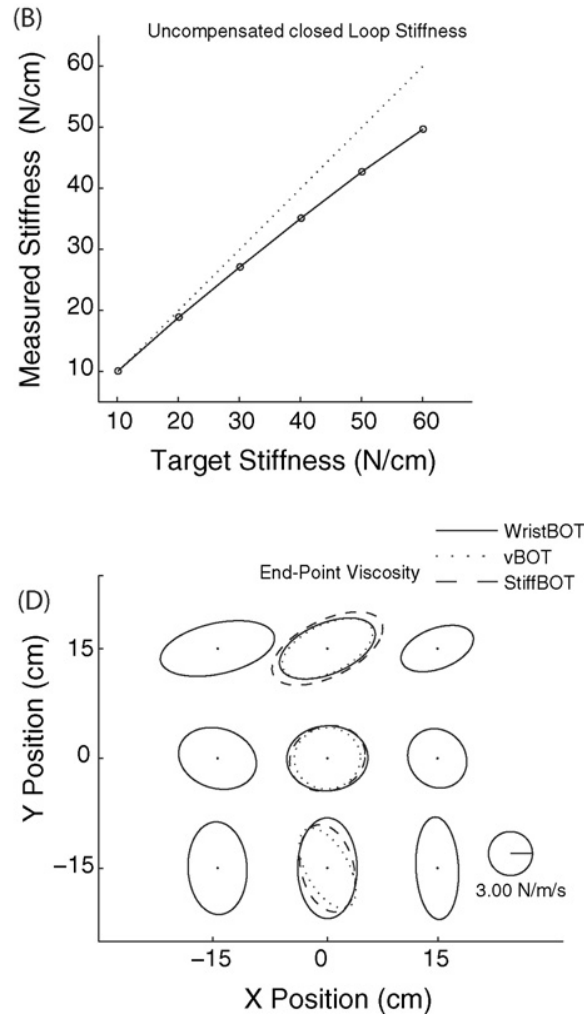

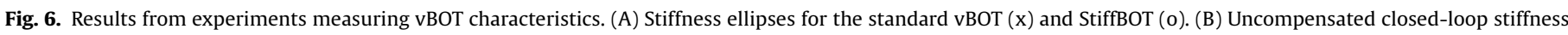

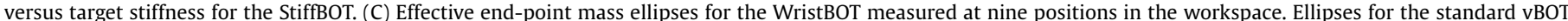

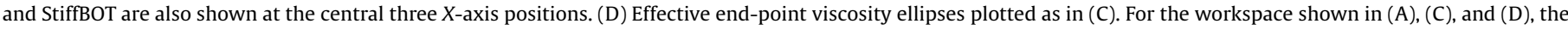

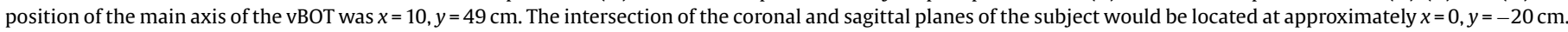

the centre of mass with respect to the handle was chosen randomly without replacement from the set $\left(0^{\circ}, 7.2^{\circ}, 14.4^{\circ}, \ldots, 352.8^{\circ}\right)$. Thus, each direction was experienced once within a block. The virtual object was presented haptically for $5 \mathrm{~s}$ after which the forces and torques were ramped linearly to 0 over $100 \mathrm{~ms}$. A tone then signalled that the response was required and subjects indicated the perceived direction of the centre of mass by making a movement towards it with the handle (Fig. 8B).

Rotating a rigid object of homogeneous density about an arbitrary axis is equivalent to rotating a spherical mass of radius $r$ on the end of a rigid lever as shown in Fig. 8A, where $r$ is scaled to be appropriate for the density of the object. The moment of inertia for an arbitrary axis of rotation is given by the parallel axis theorem:

$I_{\mathrm{Z}}=I_{\mathrm{C}}+m d^{2}$

where $I_{C}$ is the moment of inertia at the centre of mass and $d$ is the distance of the rotation axis from the centre of mass. In this case $I_{C}$ is given by:

$I_{\mathrm{C}}=\frac{2}{5} m r^{2}$

The torque associated with rotating the object is given by:

$\tau=-I_{Z} \ddot{\theta}$

The forces associated with rotating the object have two components. There is a component due to the angular acceleration of the object, given by:

$F_{\mathrm{T}}=-m d \ddot{\theta}$

which is the tangential force arising from the tangential acceleration of the mass. It is perpendicular to the hypothetical lever which joins the axis of rotation to the centre of mass. There is also a force component due to the angular velocity of the object, given by:

$F_{\mathrm{C}}=-m d \dot{\theta}^{2}$

which is the centripetal force. It is directed along the lever towards the axis of rotation. The force vector combining these two components is given by:

$F=R(\theta)\left[\begin{array}{ll}F_{\mathrm{T}} & F_{\mathrm{C}}\end{array}\right]^{T}$

where $R(\theta)$ is a $2 \times 2$ rotation matrix.

To avoid measuring angular acceleration directly, the dynamics described above were approximated by simulating a spherical mass attached to the end of a lever by a stiff spring. The spring had a translational stiffness of $5000 \mathrm{~N} / \mathrm{m}$ and a rotational stiffness of $5 \mathrm{~N} \mathrm{~m} / \mathrm{rad}$. Translation and rotation of the object causes the spring to stretch which then generates forces and torques on the handle. At the same time, these forces and torques are used to update the state of the simulated mass. A small amount of damping was included to prevent oscillations (translational damping $3 \mathrm{~N} / \mathrm{m} / \mathrm{s}$; rotational damping $0.01 \mathrm{~N} \mathrm{~m} / \mathrm{rad} / \mathrm{s}$ ). Simulations in Matlab were performed to verify that the dynamics of the object were well represented. To improve stability, the object simulation was run at $2 \mathrm{kHz}$ by performing two iterations within each cycle of the $1 \mathrm{kHz}$ control loop.

The relationship between the angle of the centre of mass of the object $\theta_{\mathrm{O}}$ and the response angle for subjects $\theta_{\mathrm{R}}$ was assessed by calculating the circular-circular T-linear association statistic (Fisher, 1993). This statistic assumes the model:

$\theta_{\mathrm{R}}=\theta_{\mathrm{O}}+\phi$

such that the response of the subject depends on the object angle with some bias given by angle $\phi$. The statistic $\rho$ gives the strength of 

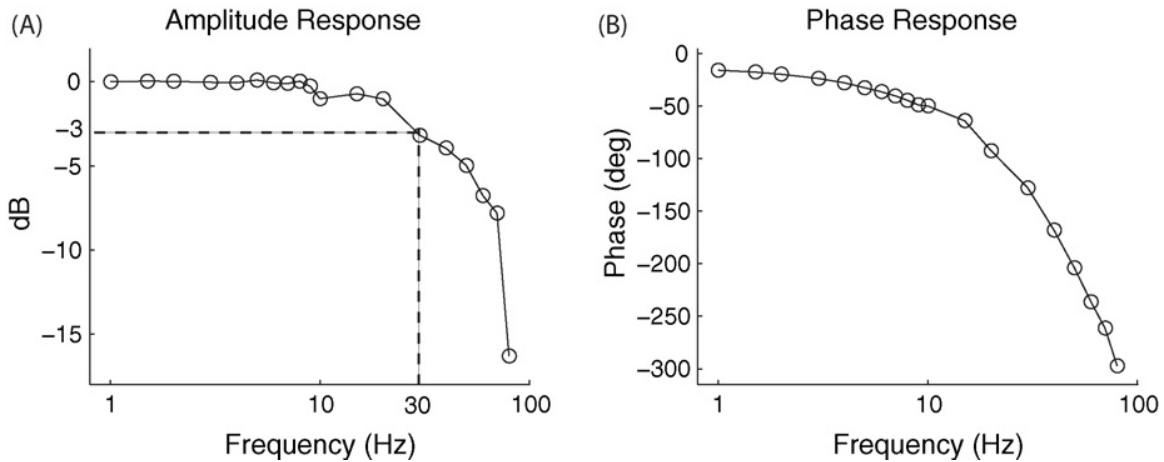

Fig. 7. Frequency response of the StiffBOT. (A) Amplitude plot showing that the response falls to $-3 \mathrm{~dB}$ at a frequency of $30 \mathrm{~Hz}$. (B) Phase plot.

association, which is 1 for perfect positive linear association and 0 when there is no association. Thus, this value represents the ability of subjects to discriminate the direction of the centre of mass based on haptic interaction with the object. The statistic was calculated for each subject and bootstrapping was used to estimate the 95\% confidence limits for the $\rho$ value for each subject.

\section{Results}

\subsection{Performance evaluation}

The measured workspace is shown in Fig. 2B. The vBOT static friction was $0.15 \pm 0.08 \mathrm{~N}$ (mean \pm SD over 30 trials) and the StiffBOT static friction was $0.20 \pm 0.08 \mathrm{~N}$ (over 30 trials). This higher value for static friction of the StiffBOT was expected due to the use of zero backlash belts which replace the low backlash units on the standard vBOT.

Fig. 6A shows the stiffness ellipses for the vBOT and StiffBOT at the centre of the workplace. It can be seen that the static stiffness at the handle was not isotropic. The stiffness ranged from 325 to $383 \mathrm{~N} / \mathrm{cm}$ for the vBOT and was higher for the StiffBOT, with values ranging from 439 to $494 \mathrm{~N} / \mathrm{cm}$.

Fig. $6 \mathrm{~B}$ shows the measured closed-loop stiffness plotted against the target stiffness for the vBOT. It can be seen that when $60 \mathrm{~N} / \mathrm{cm}$ is requested $50 \mathrm{~N} / \mathrm{cm}$ is actually achieved. This can be understood in terms of the measured stiffness of the vBOT which acts as a series compliance element with the target stiffness (that is, $1 /(1 / 60+1 / 354)=51 \mathrm{~N} / \mathrm{cm})$

Estimated effective end-point mass for the WristBOT across the workspace is shown in Fig. 6C. Values are also shown at the central
(A)
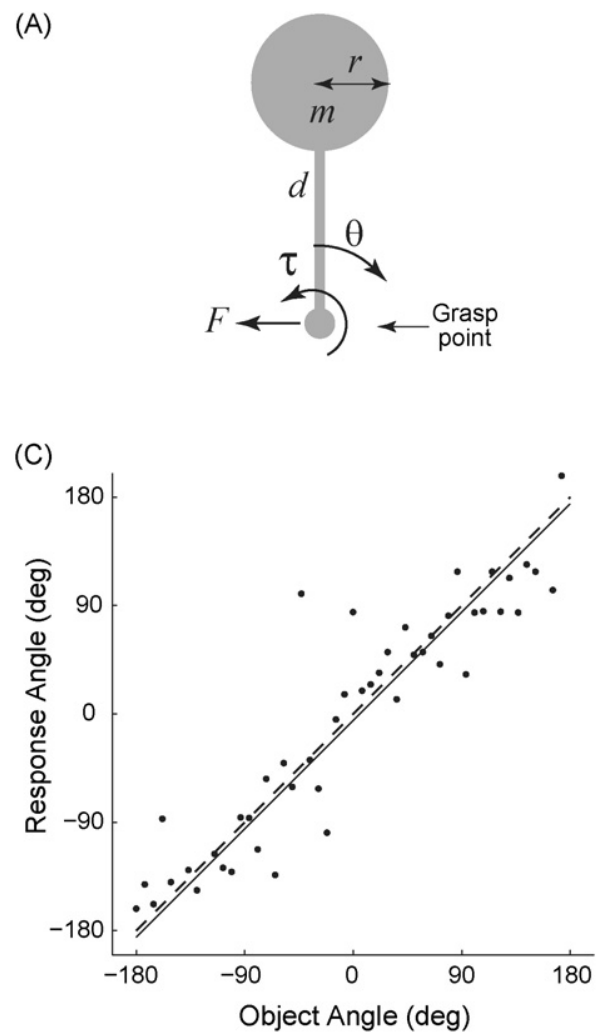

(B)

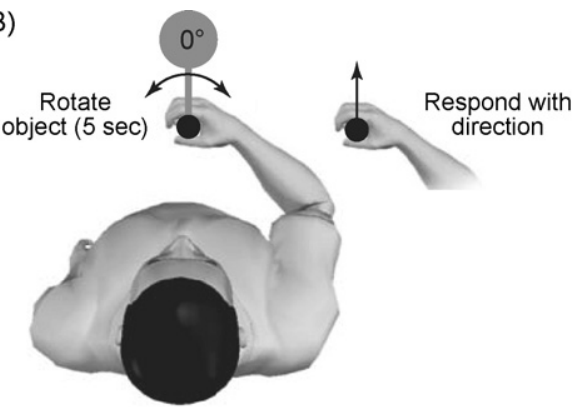

(D)

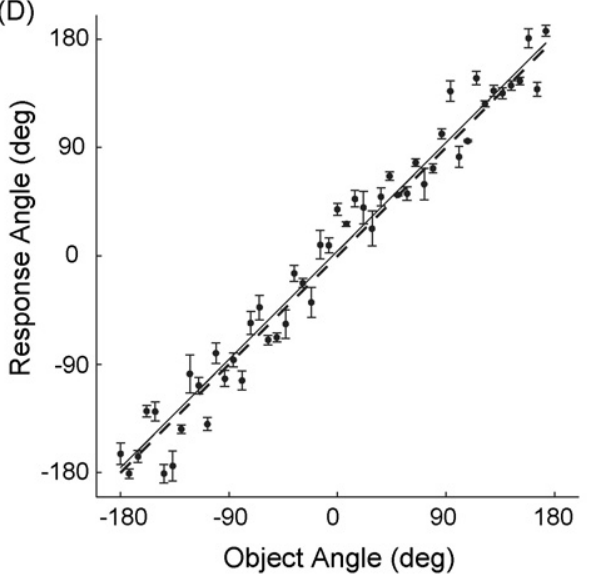

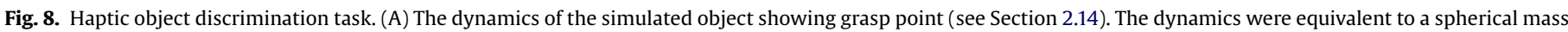

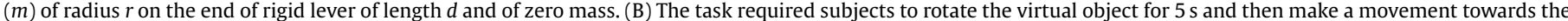

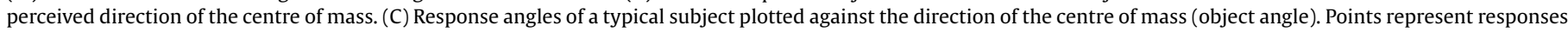

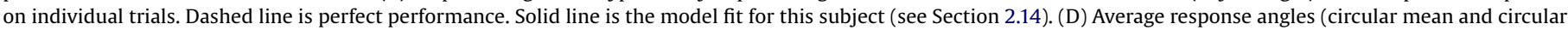
variance) across subjects (plotted as in C). 
$X$-axis locations for the vBOT and StiffBOT. The degree of anisotropy changes across the workspace and can be understood by considering the mechanical structure of the vBOT arm. For movements in the direction of the lower arm link, the effective end-point mass includes the full mass of this link. In contrast, for movements in the orthogonal direction, only part of the mass of the link contributes to the effective end-point mass.

The effective end-point mass, averaged over the three central $X$ axis positions, was quantified in terms of the values along the two principle ellipse axes. For the vBOT the range was $0.42-0.71 \mathrm{~kg}$, for the StiffBOT $0.41-0.87 \mathrm{~kg}$, and for the WristBOT $0.42-0.86 \mathrm{~kg}$. In all cases, these values include a contribution of $0.375 \mathrm{~kg}$ due to the handle, so the effective end-point mass of the arm itself is lower than that shown in the plots by this amount. As expected, adding torque control to the WristBOT increased the effective end-point mass in the direction of the lower arm link due to the addition of the torque transmission system. Similarly, the additional mass of the extra-stiff links used in the StiffBOT increased the effective end-point mass.

The estimated viscosity of the vBOT, StiffBOT and WristBOT is shown in Fig. 6D. In this case, the anisotropy can be understood in terms of the transmission system, which depends on the configuration of the arm and the movement direction. In some cases, movement of the handle drives both pulleys, whereas in others only a single pulley is involved. Clearly, the viscosity is higher in the former than the latter case.

The end-point viscosity, averaged over the three central $X$ axis positions, was also quantified in terms of the values along the two principle ellipse axes. For the vBOT the range was $3.46-5.73 \mathrm{~N} \mathrm{~s} / \mathrm{m}$, for the StiffBOT 3.95-6.51 N s/m, and for the WristBOT 3.99-6.44 N s/m. The WristBOT exhibited higher viscosity than the standard vBOT due to the extra contribution from the cable drive mechanism. Similarly, the higher viscosity of the StiffBOT was expected due to the use of zero backlash timing pulleys.

The end-point dynamic friction, averaged over the same three positions, was similarly quantified in terms of the values along two principle ellipse axes. For the vBOT the range was $0.08-0.11 \mathrm{~N}$, for the StiffBOT 0.11-0.14 N, and for the WristBOT 0.09-0.12 N. As with viscosity the WristBOT exhibited higher friction than the standard vBOT due to the extra contribution from the cable drive mechanism. Similarly, the higher friction of the StiffBOT was again expected due to the use of zero backlash timing pulleys.

The frequency response of the StiffBOT is shown in Fig. 7. It can be seen from the amplitude response (Fig. 7A) that the StiffBOT has a bandwidth of $30 \mathrm{~Hz}$.

\subsection{Object manipulation task}

There was a positive linear association between the responses of each subject and the direction of the centre of mass. Specifically, the $\rho$ values for the circular-circular T-linear association statistic (see Section 2.14) were significantly greater than zero for all subjects ( $95 \%$ confidence limits estimated by a boot-strap method). In addition, the angular offsets for all subjects except one were not significantly different from zero (two-tailed $t$-test, $p>0.05$ ). For this single subject, the angular offset was $41^{\circ}$ indicating a response bias.

The performance for a typical subject is shown in Fig. $8 \mathrm{C}$ and the mean performance across subjects is shown in Fig. 8D. These results show that subjects are able to use haptic information obtained by rotating an object to determine the direction of its centre of mass.

\section{Discussion}

\section{1. $v B O T$ design and performance}

We presented the design of a generic planar manipulandum optimized for investigation of motor learning. We discussed the design requirements for such a device and explained how they are implemented. The range of applications for the vBOT was increased by its modular designed, which supports two additional variants. In the case of the WristBOT, we implemented end-point torque control, which allows it to simulate the rotational dynamics of objects. This is a novel feature not currently available in most comparable manipulanda designs. In the case of the StiffBOT, we modified the design to make it more suitable for the measurement of arm stiffness. In addition, we described the design and construction of a controller unit, including its hardware and software interface to the host PC. Finally, we briefly discussed the accessories required to implement experimental paradigms using the vBOT. These included a mounting table, virtual reality rig, air table, and air sleds.

To quantify performance of the vBOT and its variants, we measured friction, effective end-point stiffness, mass and viscosity. Published results for existing manipulanda are limited. For example, the MIT-Manus has an end-point mass of $1 \pm 0.33 \mathrm{~kg}$, friction of $0.84 \pm 0.28 \mathrm{~N}$, and can generate a maximum end-point force of $45 \mathrm{~N}$ (Krebs et al., 1998). The manipulandum due to Acosta and colleagues is quoted as having a stiffness range of $80-660 \mathrm{~N} / \mathrm{cm}$ (Acosta et al., 2000). The haptic master has a stiffness specification of $100-500 \mathrm{~N} / \mathrm{cm}$ and minimal end-point inertia of $2 \mathrm{~kg}$ (Van der Linde et al., 2002). The StiffBOT compared well with these, exhibiting a stiffness value of $440-494 \mathrm{~N} / \mathrm{cm}$ in the centre of the workspace and an effective end-point mass ranging from 0.41 to $0.87 \mathrm{~kg}$. The bandwidth of the StiffBOT was measured to be $30 \mathrm{~Hz}$, which is much greater than that of the human arm (Kirsch and Rymer, 1992).

The addition of end-point torque control on the WristBOT resulted in a small increase in effective end-point mass and viscosity due to the pulley mechanism. Using more rigid arm sections in the StiffBOT increased its overall stiffness, as measured at the handle, over the vBOT by approximately 1.3 times. This was at the expense of a small increase in effective end-point mass and viscosity. In both cases, the increases in intrinsic dynamics were small and easily justified by the increased versatility. In the case of the WristBOT, we demonstrate its utility using an object manipulation task in which subjects were shown to correctly identify the orientation of the object based only on haptic feedback.

\subsection{Object manipulation task}

The results from our object manipulation task support previous studies, which have explored human perceptual abilities referred to as dynamic touch (Turvey, 1996). However, whereas these previous studies used real physical objects, we simulate a virtual object using the WristBOT.

In the task, subjects discriminated the direction of the centre of mass of a haptically experienced object, which is similar to previous experiments in which subjects determine the orientation of an occluded object (Pagano and Turvey, 1992; Turvey et al., 1992). The object in these previous studies was composed of long and short wooden rods configured to form an "L" shape. Subjects grasped the object at the base of the long rod and were required to indicate the direction of the short branch at the distal end. Subjects could not see the object, but were allowed to wiggle it for an unrestricted amount of time before responding. The responses closely matched the actual orientation of the distal branch (Turvey et al., 1992). In a more elaborate version of the same task, Pagano and Turvey (1992) presented subjects with a similar object which had two distal branches oriented at $90^{\circ}$. The two branches were identical and by selectively weighting one branch or the other with an attached mass, they were able to shift the orientation of the object as perceived by subjects towards the weighted branch.

The above results were explained well by a model which assumed subjects were sensitive to the orientation of the third eigenvector of the object's inertia tensor. The third eigenvector 
describes the rotational axis of an object which has the smallest moment of inertia. For the virtual object we used in our experiment, the third eigenvector points perpendicularly from the axis of rotation towards the centre of mass. However, because the WristBOT simulates object torques in only one dimension, the mechanism by which our subjects perceived the orientation of the third eigenvector could not have relied on the moment of inertia. Rather, to perform the task, our subjects had to be sensitive to the direction of the forces produced by rotating the object, which changed systematically with the different object orientations. Moreover, because they did not receive feedback about the accuracy of their responses, they had to rely on prior knowledge of object dynamics. Our results thus show that humans are sensitive to the direction of forces generated by the rotational dynamics of objects, and can use this information to perceive object orientation in the absence of cues obtained from the moment of inertia.

\subsection{Conclusions}

The standard vBOT has already been used in several published studies. The first study adopted a neuroeconomics approach which characterised the trade off between force magnitude and its duration in a virtual motor task (Kording et al., 2004). Subsequently a bimanual task using two vBOTs showed that when an action performed by one hand results in consequences at the other hand, these are represented in different coordinate frames (Bays and Wolpert, 2006). Another bimanual study showed that when each hand simultaneously learns a force field, there is neither interference nor facilitation between them (Tcheang et al., 2007). A bimanual vBOT setup has been used to examine unimanual inter-limb transfer and confirmed previous studies which showed transfer only from the dominant to the non-dominant arm (Galea et al., 2007). In a bimanual object manipulation task using the vBOT it was found that objects with simple dynamics were represented in object-based coordinates whereas those with complex dynamics were represented in arm centred coordinate. Interestingly, objects that fell between these two extremes were represented in an intermediate coordinate system (Ahmed et al., 2008). Most recently, we have shown in a bimanual object manipulation task that using the two hands together versus using them independently appears to involve partially separate representations in each case (Howard et al., 2008).

In summary, the vBOT, its variants and accessories provide a useful and versatile tool for the investigation of a wide range of issues in motor control.

\section{Acknowledgements}

We thank the Wellcome Trust and The European Project (SENSOPAC IST-2005-028056, http://www.sensopac.org) for financial support. We thank Victor Baller, Richard Symonds, Alistair Ross, John Beavis, Steven Robinson, Richard Christmas, and Dave Gautrey for their technical support.

\section{Appendix A. Supplementary data}

Supplementary data associated with this article can be found, in the online version, at doi:10.1016/j.jneumeth.2009.05.005.

\section{References}

Acosta AM, Kirsch RF, Perreault EJ. A robotic manipulator for the characterization of two-dimensional dynamic stiffness using stochastic displacement perturbations. J Neurosci Methods 2000;102:177-86.
Adelstein B. A virtual environment system for the study of human arm tremor. Cambridge: Department of Mechanical Engineering, MIT; 1989.

Ahmed AA, Wolpert DM, Flanagan JR. Flexible representations of dynamics are used in object manipulation. Curr Biol 2008;18:763-8.

Amazeen EL, Turvey MT. Weight perception and the haptic size-weight illusion are functions of the inertia tensor. J Exp Psychol 1996;22:213-32.

Bays PM, Wolpert DM. Actions and consequences in bimanual interaction are represented in different coordinate systems. J Neurosci 2006;26:7121-6.

Burdet E, Osu R, Franklin DW, Milner TE, Kawato M. The central nervous system stabilizes unstable dynamics by learning optimal impedance. Nature 2001;414:446-9.

Charnnarong J. The design of an intelligent machine for upper limb physical therapy. Cambridge: Department of Mechanical Engineering, MIT; 1991.

Criscimagna-Hemminger SE, Donchin O, Gazzaniga MS, Shadmehr R. Learned dynamics of reaching movements generalize from dominant to nondominant arm. J Neurophysiol 2003;89:168-76.

de Vlugt E, Schouten AC, van der Helm FC, Teerhuis PC, Brouwn GG. A force-controlled planar haptic device for movement control analysis of the human arm. J Neurosci Methods 2003;129:151-68.

Faye I. An impedance controlled manipulandum for human movement studies. Cambridge: Department of Mechanical Engineering, MIT; 1986.

Fisher NL. Statistical analysis of circular data. Cambridge University Press; 1993.

Franklin DW, Milner TE. Adaptive control of stiffness to stabilize hand position with large loads. Exp Brain Res 2003;152:211-20.

Galea JM, Miall RC, Woolley DG. Asymmetric interlimb transfer of concurrent adaptation to opposing dynamic forces. Exp Brain Res 2007;182:267-73.

Gibson JJ. The senses considered as perceptual systems, Boston: Houghton Mifflin; 1966 .

Gomi H, Kawato M. Equilibrium-point control hypothesis examined by measured arm stiffness during multijoint movement. Science 1996;272:117-20.

Gomi H, Kawato M. Human arm stiffness and equilibrium-point trajectory during multi-joint movement. Biol Cybern 1997;76:163-71.

Goodbody SJ, Wolpert DM. Temporal and amplitude generalization in motor learning. J Neurophysiol 1998;79:1825-38.

Hayward V, Astley OR. Performance measures for haptic interfaces. In: Giralt G, Hirzinger G, editors. Robotics research: the 7th international symposium. Springer Verlag; 1996. p. 195-207.

Hiruma K, Suzuki S, Miyazawa R, Sakashita Y, Tanaka H, Hosono K, SCARA robot, United States Patent 6212968, 1999.

Hogan N, Krebs HI. Interactive robots for neuro-rehabilitation. Restor Neurol Neurosci 2004;22:349-58.

Hogan N, Krebs HI, Charnnarong J, Srikrishna P, Sharon A. MIT-Manus: a workstation for manual therapy and training. In: IEEE international workshop on robot and human communication; 1992.

Howard IS, Ingram JN, Wolpert DM. Composition and decomposition in bimanual dynamic learning. J Neurosci 2008;28:10531-40.

Kirsch RF, Rymer WZ. Neural compensation for fatigue-induced changes in muscle stiffness during perturbations of elbow angle in human. J Neurophysiol 1992;68:449-70.

Kording KP, Fukunaga I, Howard IS, Ingram JN, Wolpert DM. A neuroeconomics approach to inferring utility functions in sensorimotor control. PLoS Biol 2004;2:e330.

Krebs HI, Hogan N, Aisen ML, Volpe BT. Robot-aided neurorehabilitation. IEEE Trans Rehabil Eng 1998;6:75-87.

Kreifeldt JG, Chuang MC. Moment of inertia: psychophysical study of an overlooked sensation. Science 1979;206:588-90.

Massie T, Salisbury JK. The PHANTOM haptic interface: a device for probing virtual objects. In: Proceedings of the ASME winter annual meeting, symposium on haptic interfaces for virtual environment and teleoperator systems, Chicago, IL; 1994.

Pagano CC, Turvey MT. Eigenvectors of the inertia tensor and perceiving the orientation of a hand-held object by dynamic touch. Percept Psychophys 1992:52:617-24.

Perreault EJ, Kirsch RF, Crago PE. Voluntary control of static endpoint stiffness during force regulation tasks. J Neurophysiol 2002;87:2808-16.

Roy J, Whitcomb LL. Comparative strucural analysis of 2-DOF semi-direct-drive linkages for robot arms. IEEE/ASME Trans Mechatron 1999;4:82-6.

Schouten AC, de Vlugt E, van Hilten JJ, van der Helm FC. Design of a torque-controlled manipulator to analyse the admittance of the wrist joint. J Neurosci Methods 2006;154:134-41.

Scott SH. Apparatus for measuring and perturbing shoulder and elbow joint positions and torques during reaching. J Neurosci Methods 1999;89:119-27.

Shadmehr R, Mussa-Ivaldi FA. Adaptive representation of dynamics during learning of a motor task. J Neurosci 1994;14:3208-24.

Solomon HY, Turvey MT. Haptically perceiving the distances reachable with handheld objects. J Exp Psychol 1988;14:404-27.

Tcheang L, Bays PM, Ingram JN, Wolpert DM. Simultaneous bimanual dynamics are learned without interference. Exp Brain Res 2007;183:17-25.

Turvey MT. Dynamic touch. Am Psychol 1996;51:1134-52.

Turvey MT, Burton G, Pagano CC, Solomon HY, Runeson S. Role of the inertia tensor in perceiving object orientation by dynamic touch. J Exp Psychol 1992;18:714-27.

Van der Linde RQ, Lammertse P, Frederiksen E. The HapticMaster, a new highperformance haptic interface. In: Proc. Eurohaptics, Edinburgh Univ.; 2002. p. $1-5$. 